Journal of Environmental
ASSN: $2525-815 \mathrm{X}$

\title{
A sucessão florestal e a arquitetura vegetal influenciam a herbivoria em Cenostigma pyramidale em Floresta Tropical Sazonal Seca?
}

\section{Do the successional stage and plant architecture influence herbivory by insects in Cenostigma pyramidale in a dry tropical forest?}

\author{
José Antônio Bezerra de Oliveira ${ }^{\mathrm{a}}$, Jarcilene Silva de Almeida ${ }^{\mathrm{b}}$
}

${ }^{a}$ Universidade Federal Rural de Pernambuco-UFRPE, Departamento de Biologia, Programa de Pós-Graduação em Ecologia, Recife-PE, Brasil. CEP: 52171-900. E-mail: j.antoniobezerra@ gmail.com.

${ }^{\mathrm{b}}$ Universidade Federal de Pernambuco-UFPE, Departamento de Botânica, Laboratório de Interações Multitróficas. Av. Prof. Moraes Rêgo s/n, Cidade Universitária, Recife-PE, Brasil. CEP: 50670-901. E-mail: jarcilene.almeida@ufpe.br.

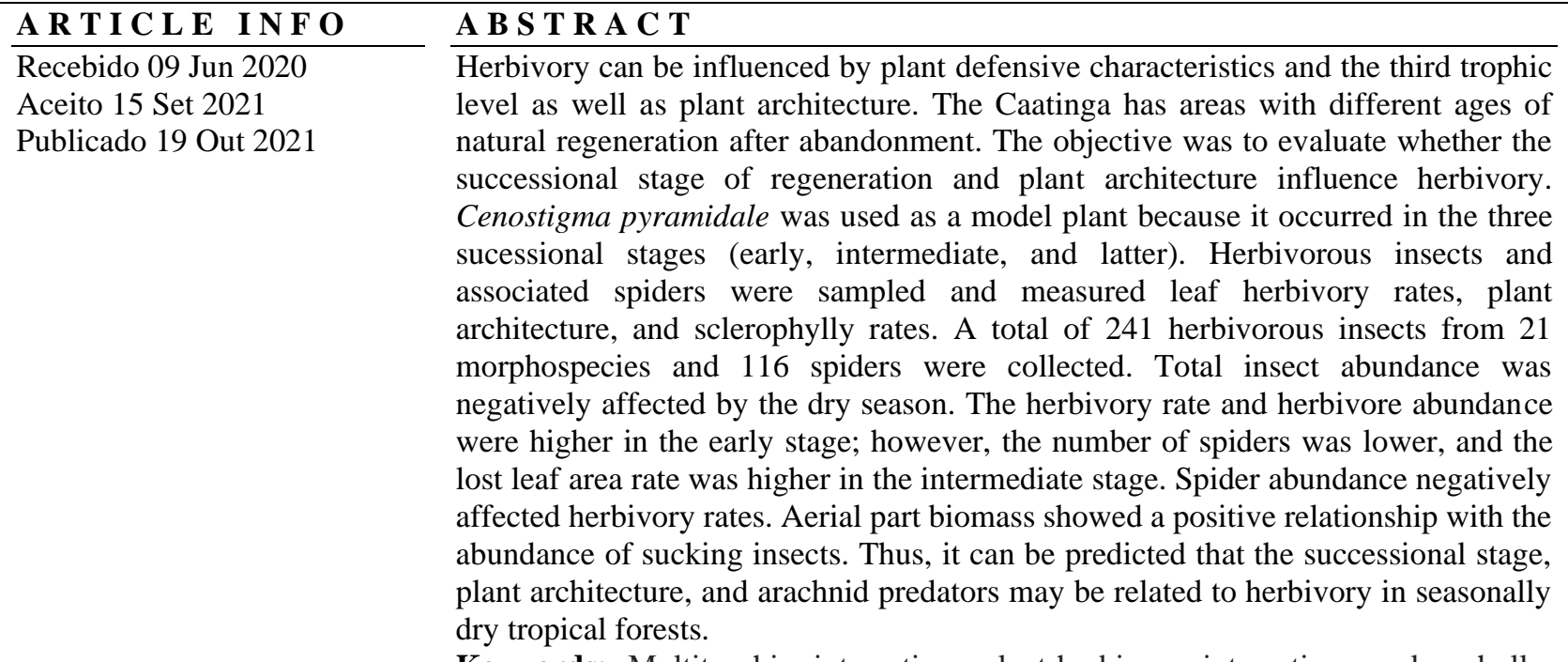

Keywords: Multitrophic interaction, plant-herbivore interactions, sclerophylly, predators

\footnotetext{
R E S U M O

A herbivoria pode ser influenciada pelas características defensivas vegetais e pelo terceiro nível trófico bem como pela arquitetura da planta. A Caatinga possui áreas com diferentes idades de regeneração natural após abandono. O objetivo foi avaliar se o estádio sucessional de regeneração e arquitetura vegetal influenciam a herbivoria. Cenostigma pyramidale foi usada como planta modelo por ocorrer nos três estádios sucessionais (inicial, intermediário e tardio). Foram amostrados insetos herbívoros e aranhas associados e mensuradas as taxas de herbivoria foliar, a arquitetura vegetal, os índices de esclerofilia. Foram coletados um total 241 insetos herbívoros de 21 morfoespécies e 116 aranhas. A abundância total de insetos foi afetada negativamente pela estação seca. A taxa de herbivoria e abundância de herbívoros foi maior no estádio inicial, no entanto o número de aranhas foi menor e taxa de área foliar perdida maior no estádio intermediário. A abundância de aranhas afetou negativamente as taxas de herbivoria. A biomassa de parte aérea apresentou uma relação positiva com a abundância de insetos sugadores. Assim, pode-se prever que o estágio sucessional, a arquitetura vegetal e predadores aracnídeos podem estar relacionados com a herbivoria em florestas tropicais sazonalmente secas.

Palavras-Chave: Interação multitrófica, interação planta-herbívoro, esclerofilia, predadores
} 


\section{Introdução}

$\mathrm{O}$ estudo das interações tróficas tem ganhado destaque nos últimos anos por apontarem processos importantes na dinâmica de populações e comunidades, sendo utilizados como subsídio para pesquisas sobre as consequências da perda de diversidade no arranjo e funcionamento das comunidades biológicas (Martins et al., 2018; Martins et al., 2019). As interações animal-planta apresentam uma diversidade e complexidade de sistemas que dificultam a elaboração de teorias gerais sobre os padrões e processos observados na natureza (Ribeiro \& Fernandes, 2000). Em especial, a relação entre plantas e seus herbívoros têm profundas implicações, tanto para a Ecologia quanto para os processos evolutivos dos mesmos (Basset, 1994), sendo a herbivoria uma importante relação que determina a dinâmica populacional, originando um acréscimo na taxa de mortalidade da população ou modificando relações competitivas entre as espécies (Bianchini \& Santos, 2005). Os insetos são os principais consumidores da produção primária terrestre (Thomazini \& Thomazini, 2000), apresentando hábito herbívoro ao menos em uma fase de seus ciclos de vida (Corrêa et al., 2008).

Neste sentido, estudos têm demonstrado que as interações multitróficas podem variar no tempo e no espaço (Martins et al., 2018). As relações de herbivoria são de grande importância para o funcionamento dos ecossistemas (Basset, 1994), sendo as condições ambientais, qualidade das plantas e presença de predadores elementos que influenciam as taxas de herbivoria (Stiling \& Moon, 2005; Aquino et al., 2017; Martins et al., 2019)

A herbivoria pode ser influenciada pelas características defensivas vegetais (controle basetopo) e pelo terceiro nível trófico, ou seja, os predadores, parasitas e/ou parasitóides dos herbívoros (controle topo-base) (Martins et al 2018). Sendo assim, as plantas desenvolveram diversas características defensivas ao longo da evolução, como a esclerofilia. Este mecanismo defensivo é comum em plantas crescidas em solos pobres em nutrientes, baixa disponibilidade de água e alta irradiação solar e confere às folhas uma alta resistência à dessecação e maior longevidade foliar (Gonçalves-Alvim et al., 2006; Silva et al., 2009). Tal condição por sua vez afeta a performance dos insetos, aumentando o tempo de desenvolvimento, diminuindo o tamanho corporal e número de ovos por fêmea, conforme registrado por Kerpel et al. (2006). A esclerofilia influencia negativamente na atividade dos insetos herbívoros (Turner, 1994; Kerpel, 2006).

A defesa vegetal biótica ocorre quando as plantas conseguem sinalizar aos determinados predadores de herbívoros, com aranha e formigas (Lima, 1997). Portanto, um estudo eficiente das interações entre plantas e insetos herbívoros deve avaliar também o terceiro nível trófico (Martins et al., 2020). As relações entre plantas e aranhas podem ser benéficas, por estes predadores reduzirem as taxas de herbivoria pela predação de herbívoros (Carter \& Rypstra, 1995; Sanders et al., 2008). Há uma íntima relação entre a estrutura trófica de uma comunidade e o nível de regeneração do ambiente (Neves, 2010; Aquino et al., 2017). Consequentemente a qualidade dos recursos disponíveis para herbívoros em florestas tropicais varia no tempo e no espaço, afetando a composição e disposição destes consumidores (Neves et al., 2014; Martins et al., 2020).

Ambientes com maior complexidade apresentam vários microhábitats, que podem se tornar abrigo contra predadores e oferecer uma variedade de recursos e um microclima propício para os herbívoros (Langellotto \& Denno, 2004; Axmacher et al., 2009).

A arquitetura vegetal é um conjunto de características estruturais da árvore (área de copa, biomassa de parte aérea, número de ramificações, altura etc), que pode ser influenciada pelo processo sucessional, gerando alta complexidade ambiental com o avanço dos estádios sucessionais (Cabral et al., 2013). Alguns estudos sugerem que a alta complexidade arquitetural vegetal, resultante do avanço sucessional, delineia uma maior abundância de predadores, intensificando assim o efeito top-down (topo-base), levando a um declínio da abundância de insetos herbívoros e, consequentemente, reduzindo a herbivoria foliar (Finke \& Denno, 2002; Langellotto \& Denno, 2004). Além do mais, Martins et al (2020a) demonstraram haver uma variação sazonal das redes de interações planta-artrópodes em florestas tropical sazonalmente seca, em particular na Caatinga, guiada pela disponibilidade hídrica (período chuvoso).

Para uma melhor compreensão das interações multitróficas e da regeneração florestal, em áreas sob ação antrópica, alguns estudos têm utilizado cronossequências de diferentes idades e locais como parâmetro para avaliar o processo de sucessão e interações associadas aos ambientes (Davis et al., 2003). Assim, o objetivo deste estudo foi avaliar se o estádio sucessional influencia a herbivoria por insetos em Cenostigma pyramidale em uma floresta tropical sazonal seca. Para isso as seguintes hipóteses foram testadas: 1) Há um aumento da riqueza e abundância de insetos herbívoros, número de aranhas e taxas de herbivoria em $C$. pyramidale com avanço do estádio sucessional, sendo estas variáveis 
influenciadas positivamente pela estação chuvosa; 2) A abundância e riqueza de herbívoros e taxa de herbivoria em $C$. pyramidale são afetadas negativamente pela abundância de aranhas $\mathrm{e}$ positivamente pela arquitetura vegetal.

\section{Material e Métodos}

\section{Área de estudo}

O estudo foi realizado em uma floresta tropical sazonal seca situada na Fazenda Tamanduá, localizada no município de Santa Terezinha, Estado da Paraíba, Região Nordeste do

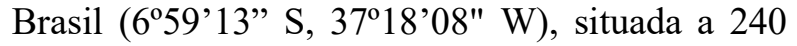
metros acima do nível do mar (Araújo, 2007). A região apresenta clima semiárido quente e de estação seca bem definida, apresentando média pluviométrica de $600 \mathrm{~mm} \cdot \mathrm{mno}^{-1}$, com chuvas irregulares e geralmente concentradas entre os meses de fevereiro e maio (Cabral et al., 2013) e o solo Neossolo Litólico predominante (Embrapa, 1999).

Presente nos três estádios sucessionais da floresta tropical seca deste estudo e sendo uma espécie de grande importância ecológica (Falcão et al., 2015, 2017; Ribeiro et al., 2021), a leguminosa Cenostigma pyramidale (Tul.) E. Gagnon \& G. P. Lewis foi a planta-modelo para o teste das hipóteses deste estudo, uma vez que apresenta ampla distribuição e estava presente em todos os estádios sucessionais (Cabral et al., 2013). A amostragem ocorreu nas populações de $C$. pyramidale inseridas em diferentes estádios de regeneração, conforme caracteriza Silva (2012): estádio sucessional inicial: área de pasto, abandonada na década 90, passando por regeneração natural há cerca de 20 anos; estádio sucessional intermediário: área de pasto ou plantio de algodão perene totalmente abandonada no final da década de 60, estando em processo de regeneração natural há mais de 45 anos; estádio sucessional tardio: área sem corte raso. Foram estabelecidas e cercadas cinco parcelas de $10 \mathrm{x}$ $50 \mathrm{~m}^{2}$ em cada estádio sucessional, impedindo a circulação de animais domésticos. A Fazenda Tamanduá adota o sistema biodinâmico, portanto não faz uso de insumos agrícolas, não é permitido a caça nem extração de madeira.

\section{Desenho amostral}

Para cada estádio sucessional, foram estabelecidas três parcelas de $1000 \mathrm{~m}^{2}(50 \mathrm{~m}$ x 20 $\mathrm{m})$ e escolhidos aleatoriamente três indivíduos de C. pyramidale, com circunferência a altura do peito de $15-38 \mathrm{~cm}$ e altura entre $2-10 \mathrm{~m}$, separados por uma distância mínima de $15 \mathrm{~m}$, tendo um total de nove indivíduos por estádio sucessional. As coletas ocorreram nos meses de maio (precipitação $=131,7$ $\mathrm{mm}^{2}$ ) e agosto (precipitação $=0,0 \mathrm{~mm}^{2}$ ) de 2014 .

Características do solo, riqueza de espécies arbóreas e arquitetura dos indivíduos de Cenostigma pyramidale

As características do solo foram descritas por Freitas et al. (2012) e Medeiros et al. (2017); e a riqueza de espécies arbóreas foi realizada por Cabral et al. (2013). Os indivíduos de $C$. pyramidale tiveram altura, diâmetro à altura do peito (DAP), área da copa e número de perfilhos mensurados. A partir do DAP, foi feito o cálculo para obtenção da biomassa da parte área, proposta por Sampaio e Silva (2005): B=0,2365 DAP 2,1928

\section{Taxas de herbivoria foliar (área foliar perdida) $e$} indice de esclerofilia

Para cada indivíduo foram aleatoriamente selecionadas quinze folhas, totalizando 135 folhas por estádio sucessional. As folhas foram digitalizadas em scanner e tiveram sua área foliar total e área foliar perdida medida pelo software ImageJ (Rasband, 1997), aplicando a Equação 01.

$\%$ Herbivoria $=($ área comprometida/área total $) \times 100$ Eq.(01)

Para a obtenção da massa foliar específica (MFE) variável que define a esclerofilia, as folhas foram mantidas em estufa por $72 \mathrm{~h}$ a $70^{\circ} \mathrm{C}$ e tiveram suas massas foliares aferidas e aplicadas à equação: MFE=Massa Foliar/Área Foliar (Turner, 1994, Madeira et al. 1998, Gonçalves-Alvim et al., 2006).

\section{Amostragem de insetos herbivoros e predadores aracnídeos}

Os insetos herbívoros de vida livre e aranhas foram amostrados a partir do método de Basset (1999) com guarda-chuva entomológico, foram realizados dez batimentos em três diferentes ramos de cada indivíduo de $C$. pyramidale. A amostragem foi feita em três diferentes horários, de modo a contemplar todas as parcelas em todos os horários. Os animais amostrados foram armazenados em etanol a 70\%, triados e separados em morfoespécies. Agrupou-se os insetos herbívoros em duas guildas: sugadores e mastigadores. Foram considerados sugadores insetos adultos e juvenis da sub-ordem Sternorryncha, Auchenorryncha e demais heterópteros herbívoros e os mastigadores, insetos adultos das ordens Orthoptera, Coleoptera e larvas da ordem Lepidoptera, segundo Gullan \& Cranston (2010), sendo identificados em nível de família. As aranhas amostradas tiveram apenas o número total mensurado por árvore. 


\section{Análise estatística}

Quando os dados se apresentaram heterogêneos, foi feita transformação para raiz quadrada e log. Para testar a hipótese 1 foi realizada análise de covariância (ANCOVA), utilizando como covariáveis a esclerofilia, biomassa de parte aérea e área da copa. Para a hipótese 2 foram realizadas regressões lineares múltiplas. As análises foram realizadas no software R ( R Development Core Team, 2008).

\section{Resultados \\ Dados biométricos de C. pyramidale}

A média de altura das árvores foi diretamente proporcional à idade dos estádios sucessionais, onde árvores mais altas foram encontradas no estádio tardio, seguida dos estádios intermediário e inicial. As árvores com maiores áreas de copa e maior biomassa da parte aérea foram às estabelecidas no estádio intermediário de sucessão. As árvores dos estádios inicial e intermediário foram as que apresentaram maior número de ramificações à altura do peito (perfilhos) (Tabela 1).

Tabela 1. Dados dos indivíduos de Cenostigma pyramidale em diferentes estádios sucessionais de regeneração de uma floresta tropical sazonal seca, Santa Terezinha, Paraíba, Brasil. Números entre parênteses representam o desvio padrão. Fonte: Oliveira \& Almeida (2021).

\begin{tabular}{ccccc}
\hline \multirow{2}{*}{ Estádio } & Altura & Área da copa & Biomassa aérea & \multirow{2}{*}{$\mathbf{N}^{\mathbf{0}}$ de perfilhos } \\
\hline Inicial & $(\mathbf{m})$ & $\left(\mathbf{m}^{\mathbf{2}}\right)$ & $\left(\mathbf{M g . h a}^{-1}\right)$ & \\
Intermediário & $2,93(0,56)$ & $12,5(4,18)$ & $6,19(2,58)$ & $12(5)$ \\
Tardio & $3,97(0,27)$ & $13,3(2,94)$ & $7,31(3,72)$ & $11(5)$ \\
\hline
\end{tabular}

Taxas de herbivoria foliar (área foliar perdida) $e$ esclerofilia

As maiores taxas de área foliar perdida foram encontradas no estádio intermediário $(7,68 \%$ e 7,36\%), seguido do estádio inicial $(6,03 \%$ e $6,36 \%)$ e tardio $(5,43 \%$ e $5,87 \%)$, na estação chuvosa e seca, respectivamente. No entanto, a taxa de área foliar perdida só apresentou diferença estatística entre os três estádios sucessionais $(\mathrm{F}=$ $5,82 ; \mathrm{p}<0,01)$.

Os índices de esclerofilia para estação chuvosa e seca foram maiores no estádio inicial $(0,57$ e 0,59$)$, tardio $(0,50$ e 0,53$)$ e intermediário $(0,46$ e 0,50$)$, respectivamente. De um modo geral, a esclerofilia teve maiores valores na estação seca em todos os estádios sucessionais.

\section{Insetos herbivoros e predadores aracnídeos}

Foram amostrados 241 insetos herbívoros, distribuídos em 21 morfoespécies. A riqueza e a abundância total de insetos herbívoros tiveram maiores valores para o estágio intermediário, inicial e tardio, respectivamente. A riqueza e abundância de mastigadores entre os estádios foram maiores que a dos herbívoros sugadores. A estação chuvosa apresentou maior riqueza e abundância de herbívoros, sendo os mastigadores a guilda mais expressiva na estação chuvosa. A família mais representativa da guilda dos mastigadores foi Curculionidae (Coleoptera), os sugadores tiveram a família Tingidae (Heteroptera) como mais abundante. Na estação seca, houve um decréscimo da riqueza de insetos herbívoros, tendo os mastigadores como a guilda com maior abundância de espécies (Tabela 2). Com relação às aranhas, foram amostradas no total 116 indivíduos, sendo o estádio inicial o que apresentou o maior número de espécimes tanto na estação chuvosa (26) quanto na seca (21), seguido do estádio intermediário $(17 ; 20)$ e estádio tardio $(19 ; 13)$.

Tabela 2. Abundância e riqueza de insetos herbívoros amostrados em indivíduos de Cenostigma pyramidale (Fabaceae) em estádios sucessionais de floresta tropical sazonal seca em maio (I) e em agosto (II) de 2014, Santa Terezinha, Paraíba, Brasil. Fonte: Oliveira \& Almeida (2021).

\begin{tabular}{|c|c|c|c|c|c|c|c|c|c|c|c|c|}
\hline \multirow{3}{*}{ Táxon } & \multicolumn{4}{|c|}{ Inicial } & \multicolumn{4}{|c|}{ Intermediário } & \multicolumn{4}{|c|}{ Tardio } \\
\hline & \multicolumn{2}{|c|}{ Abundância } & \multicolumn{2}{|c|}{ Riqueza } & \multicolumn{2}{|c|}{ Abundância } & \multicolumn{2}{|c|}{ Riqueza } & \multicolumn{2}{|c|}{ Abundância } & \multicolumn{2}{|c|}{ Riqueza } \\
\hline & I & II & I & II & I & II & $\mathbf{I}$ & II & I & II & I & II \\
\hline \multicolumn{13}{|l|}{ Coleoptera } \\
\hline Crysomelidae & & & & & & & & & & & & \\
\hline $\begin{array}{l}\text { Galerucinae sp1 } \\
\text { Curculionidae }\end{array}$ & 1 & 0 & 1 & 0 & 2 & 0 & 1 & 0 & 2 & 0 & 1 & 0 \\
\hline Curculionidae sp1 & 12 & 5 & 1 & 1 & 11 & 8 & 1 & 1 & 10 & 1 & 1 & 1 \\
\hline Curculionidae sp2 & 7 & 3 & 1 & 1 & 4 & 3 & 1 & 1 & 5 & 0 & 1 & 0 \\
\hline
\end{tabular}




\begin{tabular}{|c|c|c|c|c|c|c|c|c|c|c|c|c|}
\hline \multirow{2}{*}{\multicolumn{13}{|c|}{$\frac{\text { Heteroptera }}{\text { Tingidae }}$}} \\
\hline & & & & & & & & & & & & \\
\hline Tingidae sp1 & 6 & 3 & 1 & 1 & 9 & 3 & 1 & 1 & 4 & 1 & 1 & 1 \\
\hline \multicolumn{13}{|l|}{ Homoptera } \\
\hline \multicolumn{13}{|l|}{ Membrancidae } \\
\hline \multicolumn{13}{|l|}{ Psyllidae } \\
\hline Psyllidae sp1 & 2 & 1 & 1 & 1 & 3 & 3 & 1 & 1 & 1 & 1 & 1 & 1 \\
\hline \multicolumn{13}{|l|}{ Cicadellidade } \\
\hline Cicadellidade sp1 & 3 & 0 & 1 & 0 & 6 & 2 & 1 & 1 & 4 & 2 & 1 & 1 \\
\hline \multicolumn{13}{|l|}{ Lepidoptera } \\
\hline Larva sp1 & 4 & 0 & 1 & 0 & 2 & 0 & 1 & 0 & 1 & 0 & 1 & 0 \\
\hline Larva sp2 & 4 & 0 & 1 & 0 & 3 & 0 & 1 & 0 & 6 & 1 & 1 & 1 \\
\hline Larva sp3 & 4 & 0 & 1 & 0 & 8 & 0 & 1 & 0 & 0 & 0 & 0 & 0 \\
\hline Larva sp4 & 1 & 0 & 1 & 0 & 1 & 0 & 1 & 0 & 2 & 0 & 1 & 0 \\
\hline Larva sp5 & 2 & 0 & 1 & 0 & 0 & 0 & 0 & 0 & 3 & 0 & 1 & 0 \\
\hline \multicolumn{13}{|l|}{ Orthoptera } \\
\hline \multicolumn{13}{|l|}{ Gryllidae } \\
\hline Gryllidae sp1 & 2 & 3 & 1 & 1 & 3 & 5 & 2 & 1 & 1 & 0 & 1 & 0 \\
\hline Gryllidae sp2 & 7 & 4 & 1 & 1 & 3 & 5 & 2 & 1 & 1 & 0 & 1 & 0 \\
\hline Procospiidae & & & & & & & & & & & & \\
\hline Proscopiidae sp1 & 6 & 0 & 1 & 0 & 9 & 0 & 1 & 0 & 2 & 0 & 1 & 0 \\
\hline
\end{tabular}

Influência do estádio, estação, arquitetura $e$ predadores aracnídeos

Os resultados mostraram que apenas o número de aranhas, taxa de herbivoria e esclerofilia foram afetadas pelo estádio sucessional, sendo que o estádio intermediário apresentou menor valor para a abundância de aranhas e esclerofilia e maiores taxas de área foliar perdida. A abundância total de insetos herbívoros e a abundância de sugadores foi afetada pela estação. Os índices de esclerofilia sofreram aumento em todos os estádios da época chuvosa para a seca (Tabela 3 ).

Tabela 3. Resultados da ANCOVA para a relação das variáveis respostas com os estádios e as estações. Fonte: Oliveira \& Almeida (2021).

\begin{tabular}{lcccc}
\hline \multirow{2}{*}{ Variável Resposta } & \multicolumn{2}{c}{ Estádios } & \multicolumn{2}{c}{ Estações } \\
\cline { 2 - 5 } & $\mathbf{F}$ & $\mathbf{P}$ & $\mathbf{F}$ & $\mathbf{P}$ \\
\hline Abundância total de herbívoros & 1,06 & $>0,05$ & 6,52 & $<0,05^{*}$ \\
Riqueza total de herbívoros & 0,21 & $>0,05$ & 6,71 & $<0,05^{*}$ \\
Abundância de aranhas & 3,59 & $<0,05^{*}$ & 2,53 & $>0,05$ \\
Taxa de herbivoria & 12,9 & $<0,01^{*}$ & 1,39 & $>0,05$ \\
Esclerofilia & 21,64 & $<0,01^{*}$ & 8,94 & $<0,01^{*}$ \\
\hline
\end{tabular}

A abundância de aranhas $\left(\mathrm{F}=22,5, \mathrm{R}^{2}=\right.$ $0,29, \mathrm{P}<0,01)$ afetou a taxa de herbivoria e a biomassa de parte aérea teve relação positiva com a abundância e riqueza de herbívoros sugadores ( $\mathrm{F}$ $\left.=1,80, \mathrm{R}^{2}=0,06, \mathrm{P}<0,01\right)$.

\section{Discussão}

Dados biométricos de Cenostigma pyramidale

A maior média de altura das árvores e o menor número de ramificações no estádio tardio em relação ao estádio inicial pode estar relacionada provavelmente à idade dos indivíduos. Cada estágio sucessional contém espécies com diferentes estratégias de vida. Essas diferenças podem estar relacionadas com a plasticidade fenotípica a ambientes específicos (Endara \& Coley, 2011), entretanto, se faz necessária a análise desta característica vegetal. Falcão et al. (2015) sugerem que $C$. pyramidale pode ser considerada uma espécie importante na gestão e reflorestamento de áreas degradadas, devida sua plasticidade fenotípica. Os estádios intermediário e tardio tiveram árvores com maiores áreas de copa, sugerindo que as características do solo, como maior quantidade de água, fósforo, potássio, carbono e nitrogênio encontrado nestes estágios mais tardios (Souza et al., 2012) possam estar influenciando o desenvolvimento destes indivíduos.

Maiores valores de biomassa de parte área de indivíduos de Cenostigma pyramidale também foram encontrados por Cabral et al. (2013) para o 
estádio intermediário, em comparação ao inicial. Souza et al. (2012), investigando a influência do solo na biomassa foliar de algumas espécies arbóreas da mesma área deste estudo, encontraram maior biomassa de folhas de C. pyramidale no estádio intermediário de sucessão, em comparação com os outros estádios. Mesmo com poucos indivíduos no estádio inicial, C. pyramidale possui resistência à seca e compete por luz de maneira muito efetiva, tornando-se uma das espécies predominantes nos estádios avançados de sucessão (Aurino 2007).

\section{Insetos herbivoros e predadores aracnideos}

Em seus estudos com insetos herbívoros em florestas tropicais, Basset (1999), Campos et al. (2006), Silva et al. (2009), Neves et al. (2014) e Silva (2014) também encontraram as famílias Curculionidae, Tingidae e Psyllidae como as mais representativas entre os estádios sucessionais.

A abundância e a riqueza da guilda dos mastigadores foi superior à dos sugadores, entre os estádios de sucessão e também entre as estações. De um modo geral houve uma queda na abundância de mastigadores da estação chuvosa para a seca. Entretanto, na estação seca pode haver também maior quantidade de galhos mortos na copa, facilitando a ocorrência de coleópteros que mastigam madeira ou de dieta variada (Novotny \& Basset, 2005; Silva, 2014).

Alguns fatores como a competição e a repartição de recursos alimentares em uma determinada estação podem ser elementos importantes para, junto com os fatores climáticos, promover alterações na abundância de herbívoros (Silva et al., 2011, Freire et al., 2014). O período seco é caracterizado pela queda das folhas em ambientes sazonais, o que pode conferir baixa adaptabilidade para herbívoros de vida livre, tendo estes que migrar para hospedeiras com copa perene (Silva, 2014)

A abundância e a riqueza de sugadores praticamente não variaram entre os estádios sucessionais e entre as estações. É provável que esses insetos que se alimentam de seiva tenham uma maior especificidade com o hospedeiro que os mastigadores, sendo a qualidade do recurso um fator importante para seleção da planta (Styrsky \& Eubanks, 2007). Estudos evidenciam sincronismo entre herbívoros sugadores os aspectos fenológicos da planta hospedeira, sugerindo menor efeito do habitat para esta guilda (Silva et al., 2009).

Neves et al. (2014) relatam que os insetos herbívoros mastigadores são, de modo geral, capazes de forragear várias espécies hospedeiras se comparados com os sugadores de seiva, pois os insetos sugadores, geralmente especialistas, são influenciados pelas particularidades da planta hospedeira, enquanto os insetos herbívoros mastigadores, mais generalistas, respondem comumente aos efeitos arquiteturais da planta (Neves et al., 2014; 2015). Entretanto são necessários experimentos para analisar a preferência e o desempenho de herbívoro nos estádios sucessionais de florestas tropicais sazonais secas (Neves et al., 2014). O predomínio de ortopteroides na estação seca também foi relatado por Silva (2014) em uma floresta tropical seca do Brasil. Orthoptera e Phasmida possuem indivíduos capazes forragearem folhas de baixa qualidade e de idades avançadas, por serem herbívoros generalistas com porções do intestino mais longas e mandíbulas mais robustas (Silva, 2014).

A maior abundância de herbívoros registrada para o estádio intermediário em comparação ao inicial pode estar relacionada com a conexão entre as copas das árvores estabelecidas neste estágio, que apresenta riqueza de espécies arbóreas bem superior que a do estádio inicial (Cabral et al., 2013), favorecendo a dispersão dos insetos herbívoros de uma árvore para outra (Campos et al., 2006). É possível que a maior abundância de insetos herbívoros no estádio inicial em comparação com o tardio fora devido a menor altura e área de copa das plantas amostradas neste estádio. Logo, a densidade de insetos herbívoros pode ser maior em indivíduos estabelecidos no estádio inicial, em consequência à menor área total das árvores se comparados com os estádios avançados (Silva et al., 2011).

$\mathrm{O}$ aumento da abundância e riqueza de insetos herbívoros de acordo com a complexidade estrutural do habitat foi descrito por vários autores (Lewinsohn et al., 2005; Axmacher et al., 2009), porém não foi encontrado este padrão no presente estudo. Este estudo, assim como outros realizados em florestas tropicais (Basset et al., 1999; Campos et al., 2006; Varanda \& Pais, 2006; Silva et al., 2009; Neves et al., 2014; Silva, 2014), mostrou maior riqueza de espécies raras e pequeno número de espécies abundantes, corroborando o relatado por Price et al. (1995) para insetos herbívoros em regiões tropicais.

As aranhas estiveram presentes tanto na estação chuvosa quanto na estação seca. Porém, houve uma maior presença destes predadores no estádio inicial. Alguns estudos apontam um aumento na abundância de predadores invertebrados com o aumento complexidade ambiental, devido a um maior número de presas e microclima mais favorável (Langellotto \& Denno, 2004; Sanders et al., 2008). Este padrão não foi observado neste estudo, possivelmente porque a abundância de aranhas está fortemente 
condicionada à disponibilidade de presas, pois estes organismos são predadores que não utilizam outros recursos alternativos (Hurd \& Fagan, 1992). Se observadas as variações na abundância de aranhas e insetos sugadores entre estádios sucessionais, houve uma certa similaridade, assim como relata Silva et al. (2009). É provável que estes herbívoros, devido o hábito mais sedentário e longo tempo de forrageamento, sejam presas mais fáceis de capturar (Styrsky \& Eubanks, 2007).

\section{Taxas de herbivoria e esclerofilia}

As maiores taxas de área foliar registradas em indivíduos presentes no estádio intermediário também foram observadas por Magalhães (2011). No entanto, dois anos depois, após período de seca prolongada, Ribeiro et al (2021) não encontram diferenças significativas nas taxas de herbivoria entre os estádios, provavelmente devido ao aumento da densidade de herbívoros em todos os estádios após chuvas, pela disponibilidade do recurso planta após um período atípico. Segundo Cabral et al. (2013), a maior abundância de $C$. pyramidale no estádio intermediário indicam um maior valor de importância desta espécie neste ambiente. O papel da abundância e riqueza vegetal na caracterização da herbivoria foliar é uma das indagações biológicas mais intrigantes (Vehviläinen et al., 2007). Em outras florestas secas, Silva et al. (2012) e Neves et al. (2014) também verificaram uma maior porcentagem de herbivoria em estágios intermediários.

Em seu estudo com herbivoria em florestas de diferentes estádios sucessionais, Ernest (1989) constatou altas taxas de herbivoria em florestas secundárias com idades avançadas, quando comparadas a florestas em estágios iniciais. Florestas em diferentes estádios sucessionais apresentam uma complexidade ambiental distinta, uma vez que áreas iniciais possuem uma estrutura da comunidade vegetal mais simples e com uma menor diversidade de espécies vegetais (Lewinsohn et al., 2005; Madeira et al., 2008).

As menores taxas de herbivoria nos estádios iniciais podem estar ligadas às estratégias defensivas da planta hospedeira, onde os indivíduos estabelecidos neste ambiente provavelmente estejam sob estresse intenso ou frequente, devida a alta incidência luminosa e baixa quantidade de nutrientes do solo (Fine et al., 2006; Freitas, 2012), investindo em folhas mais resistentes, com consequente aumento da densidade foliar (Valladares \& Pugnaire, 2007 Falcão et al., 2017). Neste sentido, folhas com maior densidade mediante estresse podem ser menos consumidas por herbívoros. As folhas dos indivíduos do estádio inicial apresentaram maior índice de esclerofilia. Apesar da área foliar específica estar diretamente relacionada com a resistência ao ataque de herbívoros (Silva et al., 2009), neste estudo, esta característica isolada não explicou as taxas de herbivoria correspondentes.

Segundo Ribeiro et al (2021), as folhas expostas à alta incidência luminosa são mais resistentes e possuem maior teor de compostos fenólicos que as de folhas de sombra. Não houve a análise dos compostos fenólicos para este estudo, porém, a mesma autora, utilizando as mesmas áreas e planta, registrou para esta espécie uma concentração de compostos fenólicos nas folhas herbivoradas com tendência semelhante às taxas de herbivoria encontradas neste estudo, sugerindo que o ataque de herbívoros induz a planta a produzir estes compostos. Aquino et al., (2017) observaram uma variação nas concentrações de compostos fenólicos bem como nas taxas de herbivoria em outra espécie de caatinga, Aspidosperma pyrifolium Mart. em áreas antropizadas. Provavelmente uma concentração maior de compostos fenólicos nas folhas de indivíduos estabelecidos no estádio tardio, pode estar mais relacionada com as menores taxas de herbivoria neste ambiente, devido à idade dos indivíduos, uma vez que plantas de idade avançada alocam carboidratos para a via metabólica secundária visando a produção de composto fenólicos (Salgado et al., 2005).

Influência do estádio sucessional, estação, arquitetura vegetal e predadores aracnídeos

Apesar de não haver um aumento gradual da riqueza e abundância de insetos herbívoros, número de aranhas e taxas de herbivoria com o avanço do estádio sucessional, estas variáveis tenderam a ter influência positiva da estação chuvosa. Apenas a taxa de herbivoria apresentouse inversamente proporcional, pois geralmente é acumulativa (Silva et al., 2009; Moreira et al., 2013), onde as folhas amostradas na estação seca exibem todos os danos acumulados ao longo da estação anterior.

Três variáveis de interação multitrófica deste estudo sofreram influência do estádio sucessional. Com a diminuição do número de aranhas e menor índice de esclerofilia, houve maiores taxas de herbivoria no estádio intermediário. Porém, não houve relação entre as taxas de herbivoria dos estádios e a abundância e riqueza de insetos herbívoros. A intensidade da herbivoria em florestas secas provavelmente variam em escala espacial e temporal e estudos mais amplos são indispensáveis para a compreensão do padrão herbivoria nos gradientes sucessional e sazonal nestas formações vegetais 
(Silva et al., 2009; Neves et al., 2014). Provavelmente outros fatores estão envolvidos nas taxas de consumo foliar dos herbívoros nos estágios intermediário e tardio (Silva et al., 2009), como outros possíveis predadores não-aracnídeos (aves, lagartas de Neuroptera e parasitoides), que podem estar mais relacionados com abundância de herbívoros neste sistema (Silva et al., 2009).

A diminuição da abundância e riqueza total de herbívoros durante a estação seca parece estar relacionada com alguns fatores como competição e distribuição de alimento em determinados períodos da estação. Estes podem estar agindo sinergicamente com os elementos climáticos para delinear a abundância de insetos herbívoros (Silva et al., 2011; Freire et al., 2014). Mesmo constatando uma considerável diminuição na abundância de artrópodes durante o período seco, que é comum em ambientes tropicais (Vasconcellos et al., 2010; Freire et al., 2014), alguns estudos não mostraram este padrão (Neves et al., 2010).

A esclerofilia, que foi afetada pela estação, aumentando da época chuvosa para seca, corrobora o conceito de que maiores índices de esclerofilia na estação seca podem proteger as plantas contra as perdas de água e nutrientes, e ajudam a evitar danos causados pela alta incidência luminosa e baixa umidade (Turner, 1994; Silva et al., 2009). Logo, o excesso de luz pode causar um aumento da camada de tecido foliar, diminuindo a evapotranspiração em período mais xéricos (Turner, 1994).

A única caraterística arquitetural vegetal a influenciar a herbivoria (abundância de sugadores) foi a biomassa presente na parte aérea das plantas. Freitas \& Sampaio (2008) indicam que a biomassa aérea em áreas de caatinga está relacionada principalmente à disponibilidade de água em cada ambiente, e na maioria destes locais a biomassa oscila de 30 a 50 Mg.ha- 1. Logo, com os resultados obtidos para biomassa de parte área em Cenostigma pyramidale relacionados à biomassa total das espécies arbóreas para os mesmos ambientes deste estudo, realizados por Cabral et al. (2013), fica mais clara a importância desta espécie para esses hábitats tropicais sazonais. Assim, boa parte dos processos e interações ocorrentes nos estádios sucessionais possivelmente incluem $C$. pyramidale como elemento chave.

Por outro lado, acredita-se que as guildas de herbívoros podem agir de diferentes formas aos efeitos da área de distribuição do recurso em escalas espaciais distintas (Strong et al., 1984). Neves et al (2015) afirmam que é plausível supor que os insetos sugadores, por possuírem maior grau de monofagia, respondem à qualidade e quantidade de recursos em uma escala espacial mais local, como determinadas áreas de ramos e folhas da planta hospedeira.

Segundo Carter e Rysptra (1995), as aranhas podem reduzir taxas de herbivoria por atacarem insetos herbívoros. Assim, os resultados estatísticos indicaram que maiores taxas de herbivoria registradas no estádio intermediário podem ter relação com o menor número de aranhas, entretanto, a abundância e riqueza total de herbívoros não fora afetada pelo número destes predadores. É possível que parte das aranhas estejam forrageando nas peças florais, predando polinizadores e outros insetos (predadores, por exemplo) (Morse, 1983; Dukas \& Morse, 2003; Martins et al., 2020). Logo, observa-se que predadores de herbívoros, podem forragear de diversas maneiras e intensidades (Casula et al., 2006). Se faz necessário estudos que envolvam as interações entre aranhas e polinizadores e aranhas e herbívoros, como o relatado por Silva (2014).

\section{Conclusão}

A hipótese 1 foi parcialmente corroborada, havendo um aumento da abundância de insetos herbívoros e taxas de herbivoria do estádio inicial para o estádio intermediário. Porém, não foi observada uma continuidade para o estádio tardio, sugerindo que outros fatores podem ser mais importantes que a complexidade ambiental para a herbivoria por insetos nestes ambientes de idade mais avançada. Se faz necessária uma investigação mais minuciosa, pois boa parte das ideias e teorias para interação herbívoro-planta foram fundamentadas em estudos com florestas intermediárias e tardias, não sendo aplicadas a florestas em estádios iniciais (Silva et al., 2012). A precipitação, como esperado, influenciou positivamente as variáveis estudadas, exceto para as taxas de herbivoria, que sofreram aumento devido a acumulação de danos nas folhas com o avanço da estação. A hipótese 2 foi parcialmente corroborada, onde apenas as taxas de herbivoria podem ter sido influenciadas negativamente pelo número de aranhas. No entanto, a abundância de sugadores teve influência positiva pela variável de arquitetura vegetal biomassa de parte aérea. Portanto, pode-se sugerir que a sucessão florestal e a arquitetura vegetal influenciam a herbivoria em floresta tropical sazonal seca, porém mais estudos de longo prazo e inclusive com enfoques experimentais (Silva et al. 2012) são indispensáveis para delinear particularidades entre as interações multitróficas e variáveis ambientais dentro dos ecossistemas florestais.

\section{Agradecimentos}


Ao suporte financeiro da Fundação de Amparo a Ciência e Tecnologia de PernambucoFACEPE (APQ-0842-2.05/12); À Coordenação de Aperfeiçoamento de Pessoal de Nível SuperiorCAPES, por meio da concessão de bolsa ao primeiro autor, e ao Conselho Nacional de Desenvolvimento Científico e Tecnológico-CNPq pela concessão de Bolsa Produtividade (309965/2016-0) à Jarcilene Almeida. Ao Programa de Pós-Graduação em Ecologia da Universidade Federal Rural de Pernambuco, e ao Laboratório de Biodiversidade de Insetos da Caatinga da Universidade Federal de Campina Grande - Campus Patos, na pessoa da Dra Solange Kerpel; Ao Instituto Tamanduá pelo apoio logístico.

\section{Referências}

Aquino, R. E.; Falcão, H. M.; Almeida-Cortez, J. S. 2017. Variação nas concentrações de compostos fenólicos e nas taxas de herbivoria em Aspidosperma pyrifolium Mart. em áreas antropizadas de Caatinga. Journal of Environmental Analysis and Progress, 2, 6171.

Aurino, A. 2015. Avaliação dos impactos da extração de lenha sobre a diversidade vegetal no município de Tenório, Seridó Oriental paraibano: uma perspectiva biológica e social. Mestrado, Universidade Federal da Paraíba, 100p.

Axmacher, J.; Brehm, G.; Hemp, A.; Tünte, H.; Lyaruu, H.; Müller-Hohenstein, K.; Fiedler, K. 2009. Determinants of diversity in afrotropical herbivorous insects (Lepidoptera: Geometridae): plant diversity, vegetation structure or abiotic factors? Journal of Biogeography, 36, 337-349. doi: 10.1111/j.1365-2699.2008.01997.x

Basset, Y. 1999. Diversity and abundance of insect herbivores collected on Castanopsis acuminatissima (Fabaceae) in New Guinea: relationships with leaf production and surrounding vegetation. European Journal of Entomology, 96, 381-391.

Basset, Y. 1994. Palatability of tree foliage to chewing insects. Acta Oecologica, 15, 181191.

Cabral, G. A.; Sampaio, E. V.; Almeida-Cortez, J. 2013. Estrutura Espacial e Biomassa da Parte Aérea em Diferentes Estádios Sucessionais de Caatinga, Santa Terezinha-PB. Revista Brasileira de Geografia Física, 6, 566-574.

Campos, R.; Vasconcelos, H.; Ribeiro, S.; Neves, F.; Soares, J. 2006. Relationship between tree size and insect assemblages associated with Anadenanthera macrocarpa. Ecography, 29,
442-450. doi: 10.1111/j.2006.09067590.04520.x

Carter, P.; Rypstra, A. 1995. Top-Down Effects in Soybean Agroecosystems: Spider Density Affects Herbivore Damage. Oikos, 72, 433439. doi: $10.2307 / 3546129$

Carvalho, L.; Sebastian, N.; Araujo, H.; Dias, S.; Venticinque, E.; Brescovit, A.; Vasconcellos, A. 2015. Climatic Variables do not Directly Predict Spider Richness and Abundance in Semiarid Caatinga Vegetation, Brazil. Environmental Entomology, 4, 54-63. doi: 10.1093/ee/nvu003

Casula, P.; Wilby, A.; Thomas, M. 2006. Understanding biodiversity effects on prey in multi-enemy systems. Ecology Letters, 9, 995-1004. doi: 10.1111/j.14610248.2006.00945.x

Gullan, P.; Cranston, P.; 2010. The insects. WileyBlackwell, Chichester, 587p.

Davis, A.; van Aarde, R.; Scholtz, C.; Delport, J. 2003. Convergence Between Dung Beetle Assemblages of a Post-Mining Vegetational Chronosequence and Unmined Dune Forest. Restoration Ecology, 11, 29-42. doi: 10.1046/j.1526-100x.2003.00133.x

Dukas, R.; Morse, D. 2003. Crab spiders affect flower visitation by bees. Oikos, 101, 157163. doi: 10.1034/j.1600-0706.2003.12143.x

EMBRAPA 1999. Sistema Brasileiro de Classificação de Solos, 412p.

Endara, M.; Coley, P. 2010. The resource availability hypothesis revisited: a metaanalysis. Functional Ecology, 25, 389-398. doi: 10.1111/j.1365-2435.2010.01803.x

Ernest, K. 1989. Insect Herbivory on a Tropical Understory Tree: Effects of Leaf Age and Habitat. Biotropica, 21, 194. doi: $10.2307 / 2388642$

Falcão, H.; Medeiros, C.; Silva, B.; Sampaio, E.; Almeida-Cortez, J.; Santos, M. 2015. Phenotypic plasticity and ecophysiological strategies in a tropical dry forest chronosequence: A study case with Poincianella pyramidalis. Forest Ecology and Management, 340, 62-69. doi: 10.1016/j.foreco.2014.12.029

Falcão, H.; Medeiros, C.; Almeida-Cortez J.; Santos, M. 2017. Leaf construction cost is related to water availability in three species of different growth forms in a Brazilian tropical dry forest. Theoretical and Experimental Plant Physiology, 29, 95-108.

Fine, P.; Miller, Z.; Mesones, I.; Irazuzta, S.; Appel, H.; Stevens, M.; Sääksjärvi, I.; Schultz, J.; Coley, P. 2006 The growthdefense trade-off and habitat specialization by 
plants in Amazonian forests. Ecology, 87, S150-S162. doi: 10.1890/00129658(2006)87[150:tgtahs]2.0.co;2

Finke, D.; Denno, R. 2002. Intraguild Predation Diminished in Complex-Structured Vegetation: Implications for Prey Suppression. Ecology, 83, 643. doi: $10.2307 / 3071870$

Freire, G.; Nascimento, A.; Malinov, I.; Diniz, I. 2014. Temporal Occurrence of Two Morpho Butterflies (Lepidoptera: Nymphalidae): Influence of Weather and Food Resources. Environmental Entomology, 43, 274-282.

Gonçalves-Alvim, S.; Korndorf, G.; Fernandes, G. W. 2006. Sclerophylly in Qualea parviflora (Vochysiaceae): Influence of Herbivory, Mineral Nutrients, and Water Status. Plant Ecol, 187, 153-162. doi: 10.1007/s11258004-3286-9

Hurd, L.; Fagan, W. 1992. Cursorial spiders and succession: age or habitat structure? Oecologia, 92, 215-221. doi: 10.1007/bf00317367

Kerpel, S.; Soprano, E.; Moreira, G. 2006. Effect of nitrogen on Passiflora suberosa L. (Passifloraceae) and consequences for larval performance and oviposition in Heliconius erato phyllis (Fabricius) (Lepidoptera: Nymphalidae). Neotrop Entomol, 35, 192$200 . \quad$ doi: $10.1590 / \mathrm{s} 1519$ $566 \times 2006000200006$

Langellotto, G.; Denno, R. 2004. Responses of invertebrate natural enemies to complexstructured habitats: a meta-analytical synthesis. Oecologia, 139, 1-10. doi: 10.1007/s00442-004-1497-3

Lewinsohn, T.; Novotny, V.; Basset, Y. 2005. Insects on Plants: Diversity of Herbivore Assemblages Revisited. Annu. Rev. Ecol. Evol. Syst., 36, 597-620. doi: 10.1146/annurev.ecolsys.36.091704.175520

Lima, J. 1997. Interação de aranhas com Trichogoniopsis adenantha (D.C.) (Asteraceae). Mestrado, Universidade Estadual de Campinas, 67p.

Madeira, B. G.; Espírito-Santo, M. M.; D’Ângelo Neto, S.; Nunes, Y. R.F.; Azofeifa, A. S.; Quesada, G. W. F. M. 2009. Changes in tree and liana communities along a successional gradient in a tropical dry forest in southeastern Brazil. Plant Ecol., 201, 291-304. doi: 10.1007/s11258-009-9580-9

Martins, J. K. S. S; Oliveira, A. F. M.; Almeida, J. S. 2020. Variação sazonal das redes de interações planta-artrópodes em Floresta Tropical Sazonalmente Seca. Revista Brasileira de Geografia Física, 13, 2671-2713.
Martins, J. K. S. S; Moreira, A.; Assunção, M.; Oliveira, A.; Almeida, J. S. 2020.Trade-off in Plant-Ant Interactions: Seasonal Variations. Brazilian Journal of Biology, 80, 4, 921-933. doi: 10.1590/1519-6984.229848

Martins, J. K. S. S.; Silva, A. L. M.; Assunção, M. A. S.; Oliveira, A. F. M.; Almeida, J. S. 2020. Efeitos Indiretos de Predadores Sobre o Comportamento dos Polinizadores de Ipomoea Carnea Subs. fistulosa (Convolvulaceae) em Floresta Tropical Seca. Journal of Environmental Analysis and Progress, 5, 49-57.

Martins, J. K. S. S; Carneiro, A.; Souza, L.; Almeida-Cortez, J. S. 2019. How Pollinator Visits are Affected by Flower Damage and Ants Presence in Ipomoea carnea Subs. fistulosa (Martius and Choise) (Convolvulaceae)? Brazilian Journal of Biology, 80, 47-56.

Martins, J. K. S. S.; Souza, L. S.; Carneiro, A. G.; Almeida-Cortez, J. S. 2018. Variação Temporal e Espacial da Artropodofauna Associada a Ipomoea Carnea Subs. fistulosa (Convolvulaceae) em um Ecossistema de Floresta Tropical Seca. Journal of Environmental Analysis and Progress, 3, 356378.

Medeiros, E. V.; Duda, G. P.; Santos, L. A. R.; Lima, J. R. S.; Almeida-Cortez, J. S.; Hammecker, C.; Lardy, L.; Cournac, L. 2017. Soil organic carbon, microbial biomass and enzyme activities responses to natural regeneration in a tropical dry region in Northeast Brazil. Catena, 151, 137-146

Morse, D. 1983. Foraging patterns and time budgets of the crab spiders Xysticus emertoni Keyserling and Misumena vatia (Clerck) (Araneae: Thomisidae) on flowers. Journal Arachnology, 11, 87-94.

Neves, F.; Araújo, L.; Espírito-Santo, M.; Fagundes, M.; Fernandes, G. W.; SanchezAzofeifa, A.; Quesada, M. 2010. Canopy Herbivory and Insect Herbivore Diversity in a Dry Forest-Savanna Transition in Brazil. Biotropica, 42, 112-118. doi: 10.1111/j.17447429.2009.00541.x

Neves, F.; Silva, J.; Espírito-Santo, M.; Fernandes, G. W. 2014. Insect Herbivores and Leaf Damage along Successional and Vertical Gradients in a Tropical Dry Forest. Biotropica, 46, 14-24. doi: 10.1111/btp. 12068

Novotny, V.; Basset, Y. 2005. Host specificity of insect herbivores in tropical forests. Proceedings of the Royal Society B: Biological Sciences, 272, 1083-1090. doi: 10.1098/rspb.2004.3023 
Novotny, V.; Basset, Y.; Kitching, R. 2015. Herbivore assemblages and their food resources. In: nomes dos autores. Arthropods of tropical forests: spatio-temporal dynamics and resource use in the canopy, 1 st ed. United Kingdom, Cambridge, pp. 40-53.

Price, P.; Diniz, I.; Morais, H.; Marques, E. 1995 The Abundance of Insect Herbivore Species in the Tropics: The High Local Richness of Rare Species. Biotropica, 27, 468. doi: $10.2307 / 2388960$

Rasband, W. 1997. ImageJ. US National Institutes of Health, Maryland.

$\mathrm{R}$ development core team. 2010. R: A Language and Environment for Statistical Computing. R foundation for statistical computing, Vienna, Austria.

Ribeiro, S. P.; Fernandes, G. W. 2000. Ecologia das interações entre insetos e plantas no Cerrado: teoria e hipoteses de trabalho In: nomes dos autores, Oecologia Brasilienses: Ecologia e comportamento de insetos, 1, 299320.

Ribeiro, I. M. C.; Arruda, E. C. P.; Oliveira, A. F. M.; Almeida, J. S. 2021. Physical and chemical defenses of Cenostigma pyramidale (Fabaceae): a pioneer species in successional caatinga areas. Rev. Caatinga, 34, 2, 398-409. doi:10.1590/1983-21252021v34n216rc

Sanders, D.; Nickel, H.; Grützner, T.; Platner, C. 2008. Habitat structure mediates top-down effects of spiders and ants on herbivores. Basic and Applied Ecology, 9, 152-160. doi: 10.1016/j.baae.2007.01.003

Silva, J. 2014. Variação na diversidade de insetos e herbivoria entre grupos fenológicos de uma floresta tropical seca. Doutorado, Universidade de Brasília. 141p.

Silva, J.; Espírito-Santo, M.; Melo, G. 2011. Herbivory on Handroanthus ochraceus (Bignoniaceae) along a successional gradient in a tropical dry forest. Arthropod-Plant Interactions, 6, 45-57. doi: 10.1007/s11829011-9160-5

Silva, J.; Jesus, F.; Fagundes, M.; Fernandes, G. W. 2009. Esclerofilia, taninos e insetos herbívoros associados a Copaifera lagsdorffii Desf. (Fabaceae: Caesalpinioideae) em área de transição Cerrado-Caatinga no Brasil. Ecología Austral, 19, 197-206.
Silva, J.; Neves, F. 2014. Insect herbivores associated with an evergreen tree Goniorrhachis marginata Taub. (Leguminosae: Caesalpinioideae) in a tropical dry forest. Braz. J. Biol., 74, 623-631. doi: 10.1590/bjb.2014.0093

Silva, N.; Frizzas, M.; Oliveira, C. 2011. Seasonality in insect abundance in the "Cerrado" of Goiás State, Brazil. Rev Bras Entomol, 55, 79-87. doi: 10.1590/s008556262011000100013

Stiling, P.; Moon, D. 2004. Quality or quantity: the direct and indirect effects of host plants on herbivores and their natural enemies. Oecologia, 142, 413-420. doi: 10.1007/s00442-004-1739-4

Strong, D.; Lawton, J.; Southwood, R. 1987. Insects on Plants: Community Patterns and Mechanisms. Evolution, 41, 1137. doi: $10.2307 / 2409201$

Styrsky, J.; Eubanks, M. 2007. Ecological consequences of interactions between ants and honeydew-producing insects. Proceedings of the Royal Society B: Biological Sciences, 274, 151-164. doi: 10.1098/rspb.2006.3701

Souza, L.; Freitas, A.; Sampaio, E.; Moura, P.; Menezes, R. 2012. How much nitrogen is fixed by biological symbiosis in tropical dry forests? 1. Trees and shrubs. Nutr. Cycl. Agroecosyst., 94, 171-179. doi: 10.1007/s10705-012-9531-z

Turner, I. 1994. Sclerophylly: Primarily Protective? Functional Ecology, 8, 669-675. doi: $10.2307 / 2390225$

Varanda, E.; Pais, M. 2006 Insect folivory in Didymopanax vinosum (Apiaceae) in a vegetation mosaic of Brazilian cerrado. Braz. J. Biol., 66, 671-680. doi: 10.1590/s151969842006000400011

Vasconcellos, A.; Andreazze, R.; Almeida, A.; Araujo, H.; Oliveira, E.; Oliveira, U. 2010. Seasonality of insects in the semi-arid Caatinga of northeastern Brazil. Rev. Bras. Entomol., 54, 471-476. doi: 10.1590/s008556262010000300019

Vehviläinen, H.; Koricheva, J.; Ruohomäki, K. 2007. Tree species diversity influences herbivore abundance and damage: metaanalysis of long-term forest experiments. Oecologia, 15, 287-298. doi: 10.1007/s00442007-0673-7 